\title{
Secondary hemophagocytic lymphohistiocytosis in a child with Leptospira infection: A case report
}

\author{
Dragana Jevtic ${ }^{1}$, Dragomir Djokic ${ }^{1}$, Danka Redzic ${ }^{1}$, Dragana Aleksic ${ }^{1}$, Miro Parezanovic ${ }^{2}$, \\ Srdjan Pasic ${ }^{3}$ \\ Departments of ${ }^{1}$ Hemato-Oncology II and ${ }^{3}$ Immunology, ${ }^{2}$ Mother and Child Health Care Institute of Serbia, Radoja Dakica \\ St., Belgrade, Serbia. E-mail: drjevtic02@gmail.com \\ Received: 1st August 2017, Accepted: 30th October 2017
}

SUMMARY: Jevtic D, Djokic D, Redzic D, Aleksic D, Parezanovic M, Pasic S. Secondary hemophagocytic lymphohistiocytosis in a child with Leptospira infection: A case report. Turk J Pediatr 2018; 60: 735-738.

Leptospirosis caused by spirochetes of the genus Leptospira in most patients result in very mild illness without jaundice. However, a small portion of patients develop various complications due to the involvement of multiple organ systems. Hemophagocytic lymphohistiocytosis is characterized by prolonged fever, hepatosplenomegaly and cytopenias, hyperferritinemia and hypertriglyceridemias, hyperfibrinogenemia, and hemophagocytosis in bone marrow, lymph nodes, spleen, or liver. Hemophagocytic lymphohistiocytosis associated with leptospirosis is a very rare condition and it should be considered in patients with multiple organ dysfunctions, together with adequate laboratory findings. It can delay the correct diagnosis of leptospirosis and contribute to an adverse outcome. We present a 13-year-old girl with secondary hemophagocytic lymphohistiocytosis caused by leptospira infection and favorable outcome with appropriate antibiotics and corticosteroid therapy.

Key words: leptospirosis, hemophagocytic lymphohistiocytosis, multiorgan failure.

Leptospirosis is a common zoonosis of worldwide distribution, which predominantly involves tropical and rural areas. It is caused by pathogenic spirochetes of the genus Leptospira and is most often transmitted to humans through soil or water, contaminated with infected animal urine. ${ }^{1}$ Leptospiral infections in most patients cause a very mild sickness without jaundice. However, a small portion of patients develop various complications of multiple organ systems function, presenting as a febrile illness with icterus, splenomegaly and nephritis (Weil's disease), severe muscle pain, subconjunctival and pulmonary hemorrhages, cardiac arrhythmias and meningitis. ${ }^{2}$

Hemophagocytic lymphohistiocytosis (HLH) is a possibly lethal hyperinflammatory disorder that is characterized by histiocyte proliferation and hemophagocytosis. The most characteristic apparent signs and symptoms are fever, hepatosplenomegaly, and cytopenias. ${ }^{3}$ The diagnostic criteria of HLH are fever, hepatosplenomegaly, cytopenia affecting at least two series, ferritin above $500 \mu \mathrm{g} / \mathrm{L}$, decreased natural killer cell activity, elevated soluble CD25 level above $2,400 \mathrm{U} / \mathrm{ml}$, fasting triglyceride exceeding $265 \mathrm{mg} / \mathrm{dl}$ or fibrinogen below $150 \mathrm{mg} / \mathrm{dl}$ and hemophagocytosis in the bone marrow, spleen or lymph nodes. The diagnosis of HLH needs the existence of at least five of the standards stated above and it can be categorized as primary and secondary HLH (sHLH). ${ }^{4}$ In both of these entities hemophagocytosis is induced by cytokine storm and excessive macrophage activation. While primary HLH is, in most cases, manifested during the first year of life, the onset of sHLH can take place at any life time and is most commonly associated with Epstein-Barr virus (EBV), cytomegalovirus (CMV) and Leishmania. ${ }^{5}$ Although zoonotic diseases are leading causes of $\mathrm{HLH}$, leptospirosis associated HLH has been rarely reported in the literature ${ }^{6}$. While different entities, HLH and leptospirosis share some clinical signs, such as fever and hepatosplenomegaly. So, sHLH can delay the correct diagnosis of leptospirosis and always contribute to an adverse outcome. In this report, we present our experience with 
patient with leptospirosis associated sHLH.

\section{Case Report}

A 13-year-old girl was referred to our Institute for prolonged fever of unknown origin, macular rash, sterile pyuria, pleural effusions and hepatosplenomegaly.

The symptoms appeared 20 days before hospital admittance in a form of fever, swelling and occasional neck pain, followed by skin rash. The girl was admitted to the local hospital, where initial blood count and biochemical analysis were within normal ranges and C-reactive protein (CRP) was $36 \mathrm{mg} / \mathrm{L}$. Broad-spectrum antibiotics were initiated. In the next several days, in spite of the treatment, her overall situation was deteriorated and control laboratory findings revealed thrombocytopenia $(103,000 /$ $\mathrm{mm}^{3}$ ) and elevated aspartate aminotransferase (AST), alanine aminotransferase (ALT), lactate dehydrogenase (LDH) and CRP levels which were $469 \mathrm{IU} / \mathrm{L}, 340 \mathrm{IU} / \mathrm{L}, 1643 \mathrm{IU} / \mathrm{L}, 101$ $\mathrm{mg} / \mathrm{L}$, respectively. The abdominal ultrasound revealed hepatosplenomegaly, which was confirmed on a computerized tomography (CT) scan, alongside with growing axillar lymphadenopathy and bilateral pleural effusions. Echocardiography revealed minor pericardial effusion. Bone marrow aspiration showed 2nd degree cellularity, $\mathrm{M} / \mathrm{E}$ ratio of 3.1 to 1 ; cells in each lineage of normal morphology and of orderly and complete maturation. Serological tests performed for EBV, CMV, Herpes simplex virus were all negative. Complement components C3 and C4 levels were within normal ranges along with her rheumatoid factor level. HBs antigen anti-HBC and anti-HCV antibody, antinuclear antibodies (ANA) and anti-neutrophil cytoplasmic antibodies (ANCA) were all negative.

At admission, her vital signs were as follows: arterial blood pressure $93 / 50 \mathrm{mmHg}$, heart rate 108 beats/min., respiration rate 22 breaths/min. Physical examination revealed hyperemia of the pharyngeal wall and vesicles on the palate surface, bilaterally decreased breathing sound in the basal parts of the lungs with left-sided late inspiratory crackles, hepatosplenomegaly (liver and spleen $2 \mathrm{~cm}$ and $1 \mathrm{~cm}$ below the costal margin, respectively). The girl also had malar erythema, diffuse macular rash and pretibial edema. Other physical findings were unremarkable. Laboratory findings showed slight anemia, thrombocytopenia and neutrophilia (Table I). Biochemical analysis showed elevated levels of inflammatory markers and signs of liver and kidney insufficiency (Table I). Ferritin level was above 15,000 $\mu \mathrm{g} / \mathrm{L}$. Meropenem and cefuroxime treatment was initiated. Due to persistent oliguria, our patient was treated by continuous venovenous hemofiltration for 18 hours. The abdominal ultrasound showed existing hepatosplenomegaly, with enlarged hilar lymph nodes, bilateral pleural effusions and bilateral hyper echogenic renal parenchyma (grade 2-3). Levels of ANA, cytoplasmic and perinuclear ANCA, antidouble stranded DNA antibodies, components C3 and C4 levels and immunoglobulin levels were all in regular ranges. Serological tests performed for brucella, Hantaviruses, HIV and leptospira were all negative. In the following days biochemical analysis showed further liver enzyme elevation. Biochemical urine analysis showed elevated beta2-microglobulin which was indicative of tubulointerstitial nephritis. Control complete blood count showed further deterioration (hemoglobin $9.4 \mathrm{~g} / \mathrm{d}$, thrombocyte $\left.63,000 / \mathrm{mm}^{3}\right)$. Due to noticed disseminated intravascular coagulation, low molecular weight heparin treatment was initiated, continually with other symptomatic therapy. After a few days microscopic agglutination test (MAT) for leptospirosis came back positive (titer 1: 200), and benzyl penicillin treatment was added to previous therapy. Repeated bone marrow aspiration showed hemophagocytosis and, on the base of clinical examination and laboratory findings with soluble interleukin-2R $\alpha$ (CD25) was $3,335 \mathrm{U} / \mathrm{ml}$, secondary hemophagocytic lymphohistiocytosis was diagnosed. At first, the patient received intravenous immunoglobulin, in a dose of $1 \mathrm{~g} / \mathrm{kg}$, but the expected results were not noticed. After that, we started treatment according to modified LCH III 2002 protocol, and treated the patient with corticosteroids $\left(10 \mathrm{mg} / \mathrm{m}^{2}\right)$, with a further correction of disseminated intravascular coagulopathy and antimicrobial therapy. In the next 7 days clinical improvement was noticed gradually, with the slow remission of laboratory and radiographic findings. Control MAT performed after two weeks was negative. We proceeded with the same treatment, and after 35 days in hospital and four weeks of corticosteroid therapy, the 
girl was discharged and referred to ambulatory care, with tapering corticosteroid doses. She was regularly checked in our outpatient clinic and, she is without any sign of illness 2 years after discharge.

An informed consent was obtained from the patient and her parents for publishing this case report.

\section{Discussion}

Leptospirosis is a universal zoonosis caused by pathogenic spirochetes of the genus Leptospira. The primary source of leptospires is the excretory animal - rodents and small marsupials, cattle, pigs and $\operatorname{dog} s^{14}$, from whose renal tubules leptospires are excreted into the environment with the animal urine. There are two ways of transmission: direct and indirect. Direct transmission ensues when bacteria from tissues, body fluids or urine of truly infected or asymptomatic carrier animals enter the body of the novel host and initiate infection. It is common amongst the occupational groups who handle animals and animal tissue (veterinarians, butchers etc.). The other and the most common indirect transmission, arises when an animal or human acquires leptospirosis from the environment, contaminated with the infected animal urine. The most common entrance is through microscopic skin injuries, intact mucous membranes or peroral. ${ }^{2}$ Human-tohuman transmission through breastfeeding has similarly been recorded. ${ }^{7}$

Beside clinical presentation, diagnosis of leptospirosis is based on laboratory tests which are broadly classified into direct isolation of the organism, dark field microscopy, PCR and indirect microscopic agglutination test (MAT), which is the reference standard test and enzyme-linked immunosorbent assay (ELISA). ${ }^{2}$

There are two divergent stages of leptospiral infection: septicemia and the immune phase First being due to a vascular injury and second owing to deposition of immune complexes which lead to endothelial cell damage. ${ }^{8}$ Most cases of leptospiral infections are either subclinical or result in very mild illness, but a small number of patients develop various complications. In those patients, clinical presentation depends on predominant organs involved. Most common symptoms are skin 
rash, meningitis, uveitis, kidney and liver dysfunction, hepatosplenomegaly, jaundice, lymphadenomegaly and hemorrhage. ${ }^{2}$ The most shared hematological indicator in severe forms of leptospirosis is thrombocytopenia. ${ }^{9}$

In the literature, reports related to leptospirosis accompanied by hematological disorders are limited. We found only two case reports describing a leptospira associated HLH in children age 4 and 8.5 years. ${ }^{10,11}$ Our patient is the first child with this entity in Serbia.

After the serological test for leptospirosis came back positive, we treated our patient with antimicrobial therapy and since there was no improvement, she also received IVIG. After the diagnosis of sHLH we started treatment according to modified LCH III 2002 protocol. The patient was treated with corticosteroids and since the major improvement in her condition was achieved, it was decided not to include cyclosporine A and etoposide in the therapy. Hematological disorders subsided throughout next two weeks of treatment, after which she was discharged to ambulatory care with tapering doses of corticosteroids.

The hemophagocytosis in non-viral infections is the consequence of inappropriate cytotoxic $\mathrm{T}$ cell and natural killer cell function, overproduction of activating cytokines, such as TNF- $\alpha$ and $\gamma$ interferon and subsequent chronic stimulation of inflammatory response. The treatment of sHLH depends on its cause and the infectious agent should be eradicated promptly. Elevated cytokine levels cause hyper inflammation which should be treated with corticosteroids or immunosuppressive drugs, in order to suppress cytokine release and control cell propagation. Corticosteroids are cytotoxic to lymphocytes and suppress the expression of cytokines. Beside them, IVIG, etoposide, cyclosporine A and antithymocyte globulin (ATG), alone or combined, are other treatment choices. ${ }^{4,12,13}$ We used IVIG and corticosteroids in our patient, besides specific antimicrobial therapy.

Leptospirosis is rarely described as a cause of sHLH. Therefore, in patients with confirmed leptospirosis who present with multiple organ dysfunctions and with laboratory findings of hyperferritinemia, hypertriglyceridemia and hypofibrinogenemia, additional diagnostics should be performed to either confirm or rule out the development of sHLH.

\section{REFERENCES}

1. Yang HY, Yen TH, Lin CY, et al. Early identification of leptospirosis as an ignored cause of multiple organ dysfunction syndrome. Shock 2012; 38: 24-29.

2. Vijayachari P, Sugunan AP, Shriram AN. Leptospirosis: An emerging global public health problem. J Biosci 2008; 33: 557-569.

3. Atteritano M, David A, Bagnato G, et al. Haemophagocytic syndrome in rheumatic patients. A systematic review. Eur Rev Med Pharmacol Sci 2012; 16: 1414-1424.

4. Henter J, Horne A, Aricó M, et al. HLH-2004: Diagnostic and therapeutic guidelines for hemophagocytic lymphohistiocytosis. Pediatr Blood Cancer 2007; 48: 124-131.

5. Larroche C, Mouthon L. Pathogenesis of hemophagocytic syndrome (HPS). Autoimmun Rev 2004; 3: 69-75.

6. Cascio A, Pernice LM, Barberi G, et al. ndary hemophagocytic lymphohistiocytosis in zoonoses. A systematic review. Eur Rev Med Pharmacol Sci 2012; 16: 1324-1337.

7. Bolin C, Koellner P. Human-to-human Transmission of Leptospira interrogans by milk. J Infect Dis 1988; 158: 246-247.

8. Speelman Peter. Leptospirosis. In: Braunwald E, Fauci AS, Kasper DL, Hauser SL, Longo DL, Jameson JL (editors). Harrison's Principles of Internal Medicine (15 $5^{\text {th }}$ ed). New York: McGraw-Hill Publication, 2001: 1055-1057.

9. Wang LS, Wang CC, Huang SH, et al. Leptospirosis with transient paraparesis and thrombocytopenia: A case report. J Microbiol Immunol Infect 2012; 45: 75-78.

10. Krishnamurthy S, Mahadevan S, Mandal J, Basu D. Leptospirosis in association with hemophagocytic syndrome: A rare presentation. Indian J Pediatr 2012; 80: 524-525.

11. Sripanidkulchai R, Lumbiganon P. Etiology of obscure fever in children at a university hospital in northeast Thailand. Southeast Asian J Trop Med Public Health 2005; 36: 1243-1246.

12. Yaman Y, Gözmen S, Özkaya AK, et al. Secondary hemophagocytic lymphohistiocytosis in children with brucellosis: Report of three cases. J Infect Dev Ctries 2015; 9: 1172-1176.

13. Ishii E. Hemophagocytic lymphohistiocytosis in children: Pathogenesis and treatment. Front Pediatr 2016; 4: 47.

14. Adler B, de la Peña Moctezuma A. Leptospira and leptospirosis. Vet Microbiol 2010; 140: 287-296. 